\title{
EMULATION IN MANUFACTURING ENGINEERING PROCESSES
}

\author{
Hironori Hibino \\ Technical Research Institute \\ JSPMI \\ (Japan Societv for the Promotion of Machine Industrv) \\ 1-1-12, Hachiman, Higashikurume, Tokyo, JAPAN
}

\author{
Systems Design Dept. \\ Hosei University \\ 2-17-1, Fujimi, Chiyoda, Tokyo, JAPAN
}

\begin{abstract}
In our research, the manufacturing system emulation technology is proposed as one of the frontloading methods in the manufacturing system implementation phase. In this paper, the roles of the manufacturing system emulation technology in manufacturing engineering processes are summarized based on our analysis for the typical manufacturing engineering processes. The manufacturing system emulation environment (MSEE) to implement the manufacturing system emulation is proposed and developed. MSEE consists of our developed manufacturing cell emulator, our developed soft-wiring system, and the industrial network middleware which is one of the semi-standard middlewares. The validation of our proposed environment was carried out through a case study.
\end{abstract}

\section{INTRODUCTION}

Industry needs to design and make new products for the market in rapid succession, as it is becoming harder to keep the high value of a product in the market as a long seller. It is important for manufacturing systems to be established with flexibility, scalability and reconfigurability (Mehrabi, Ulsoy, and Koren 2000; Molina, Rodriguez, Ahuett, Cortes, Ramirez, Jimenez, and Martinez 2005). It is also important to reduce the lead-time for manufacturing engineering processes from the manufacturing system design phase to the manufacturing system implementation phase (Hibino and Fukuda 2006; Hibino, Inukai, and Fukuda 2006; Fukuda 2003). One of the solutions to realize these requirements is the front-loading method, which finds problems in advance and solves the problems at an earlier phase in the manufacturing engineering processes while limiting wasteful periods to the minimum by reducing the number of times needed to go back and refine the design (Hibino 2007). The front-loading method using simulation technologies has attracted the attention of industries with the advance of technologies such as computer calculation performance and three-dimensional CAD in- formation technologies (Hibino 2007). However as there are many limitations concerning simulation applications for many evaluation activities in the manufacturing engineering processes, it is necessary to extend the range of simulation applications through the manufacturing engineering processes. Particularly, industries need simulation technology advances in the manufacturing system implementations phase.

In our research, the manufacturing system emulation technology is proposed as one of the frontloading methods in the manufacturing system implementation phase. In this paper, the roles of the manufacturing system emulation technology in manufacturing engineering processes are summarized based on our analysis for typical manufacturing engineering processes. The manufacturing system emulation environment (MSEE) to implement the manufacturing system emulation is proposed and developed. MSEE consists of our developed manufacturing cell emulator, our developed soft-wiring system, and the industrial network middleware which is one of the semi-standard middlewares The validation of our proposed environment was carried out through a case study.

\section{THE EMULATION IN MANUFACTURING ENGINEERING PROCESS}

Firstly, the terms of manufacturing system simulation, manufacturing system emulator, and manufacturing system emulation are defined as follows.

1. Manufacturing system simulation means to create certain conditions of manufacturing systems by means of models. One of the main purposes of the manufacturing system simulation is to evaluate materials flow and information flow. A simulation is executed while paying attention to particular events of interest which occur at an instant, such as equipment start, equipment stop and so on. Figure 1 shows an outline of the manufacturing system simulation. 


\section{Hibino and Fukuda}

2. A manufacturing system emulator is a device or piece of software that enables a program or an item of equipment intended for one type of computer or equipment to be used with exactly the same results with another type of computer or equipment.

3. Manufacturing system emulation means that under a condition where parts of equipment, control programs, and manufacturing management applications are not provided in a manufacturing system, a manufacturing system operates by mixing and synchronizing the manufacturing system emulators, real equipment, real controllers, and management applications. Figure 2 shows an outline of the manufacturing system emulation.

Based on our analysis for the typical manufacturing engineering processes, manufacturing systems are established through four phases. Figure 3 shows the typical manufacturing engineering processes.

Phase 1: This phase is the planning phase to define fundamental manufacturing requirements such as target production volumes, location and so on.

Phase 2: This phase is the manufacturing system design phase to fix manufacturing specifications such as the numbers of equipment needed, layout, manufacturing management as with the Kanban system and so on.

Phase 3-1: This is a period prior to the manufacturing system implementation phase. In this period, engineers implement hardware such as special machines, transfer machines, and software such as ladder programs, robot programs, operation panels, and production control programs.

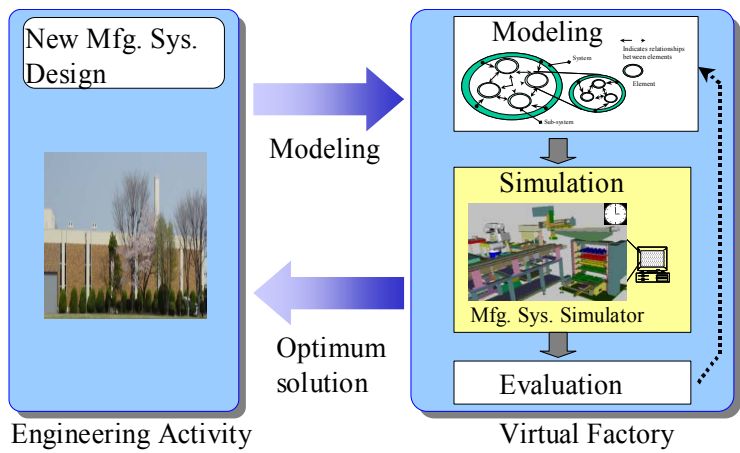

Figure 1: Manufacturing System Simulation

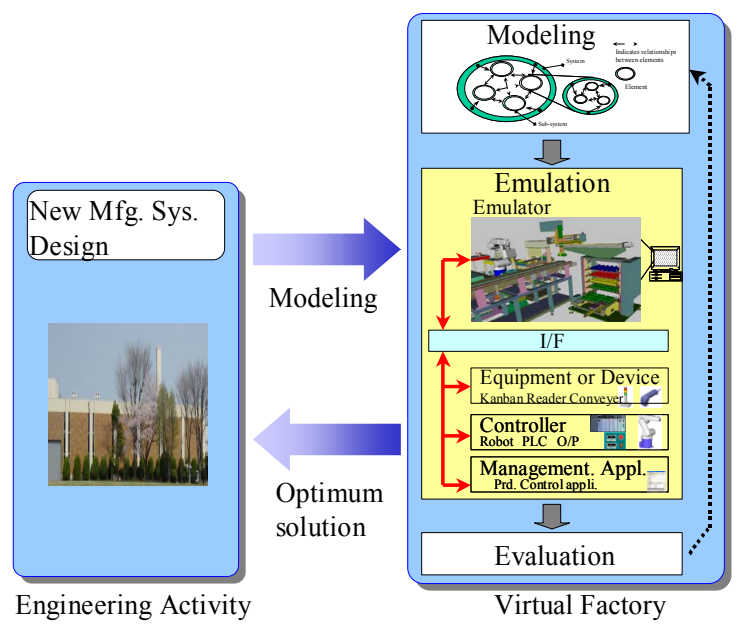

Figure 2: Manufacturing System Emulation

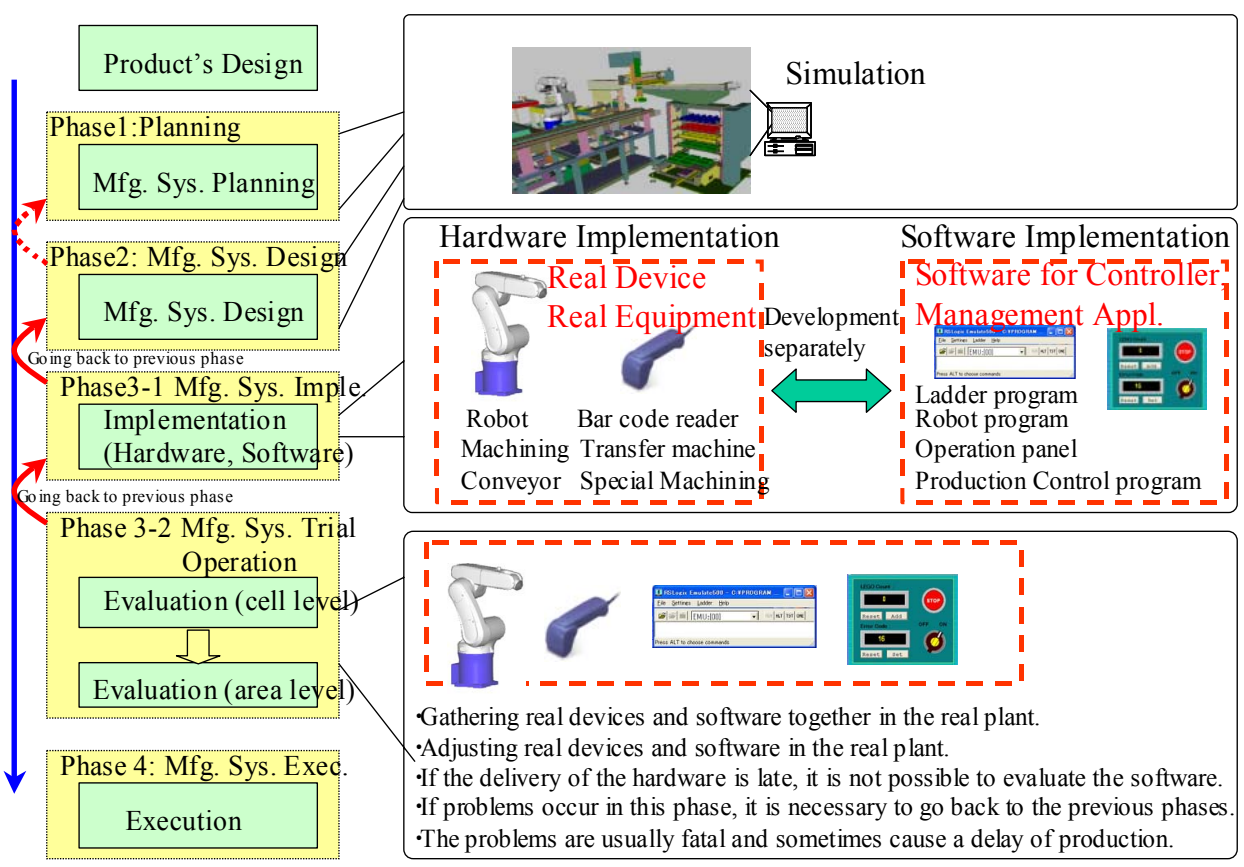

Figure 3: Typical Manufacturing Engineering Process 


\section{Hibino and Fukuda}

Phase 3-2: This is a later period of the manufacturing system implementation phase. In this period, in order to undertake the manufacturing system trial operations, engineers partially operate hardware execution and software execution and accurately evaluate their executions from the viewpoints of the manufacturing systems.

Phase 4: This phase is the actual manufacturing system execution phase.

In phase 1 and phase 2, a manufacturing system simulator plays an important role in designing and evaluating manufacturing systems by using a virtual factory model (Hibino, Fukuda, Fujii, Kojima, Mitsuyuki, and Yura 1999; Hibino, Fukuda, Yura, Mitsuyuki, and Kaneda 2002; Mitsuyuki, Kojima, Douba, Fukuda, and Arai 2004; Williams and Celik 1998).

In phase 3-1, engineers separately develop hardware such as robots and special machining devices, and software such as ladder programs and production control programs. Then they independently evaluate their developed hardware or software in their place. In phase 3-2, the developed hardware or software is gathered together and adjusted in a real plant. If the delivery of the hardware is late, it is not possible to evaluate the software. If problems occur in this phase, it is necessary to go back to the previous phases. The problems are usually fatal and sometimes cause a delay of production.
Therefore as industries need suitable simulation technology advances in this phase, methods to partially operate hardware execution and software execution and to accurately evaluate their executions from the viewpoints of manufacturing systems have not been developed. For example, methods to evaluate the facility control programs for equipment, such as ladder programs for PLC, while mixing and synchronizing real equipment and virtual factory models on the computers have not been developed. This difficulty has hindered precise and rapid support of a manufacturing engineering process. Consequently the leadtime is not reduced. To solve this difficulty, it is necessary to develop manufacturing system emulation. Using manufacturing system simulation and manufacturing system emulation, each phase can be evaluated in advance as preevaluation. Figure 4 shows the proposed manufacturing engineering processes using the manufacturing system simulation and the manufacturing system emulation.

\section{THE MANUFACTURING CELL EMULATION ENVIRONMENT}

To realize the manufacturing system emulation, we propose a manufacturing cell emulation environment (MCEE). Under a condition where part of the equipment, control programs, and manufacturing management applications are not provided in manufacturing system,

Pre-evaluation using Simulation and Emulation
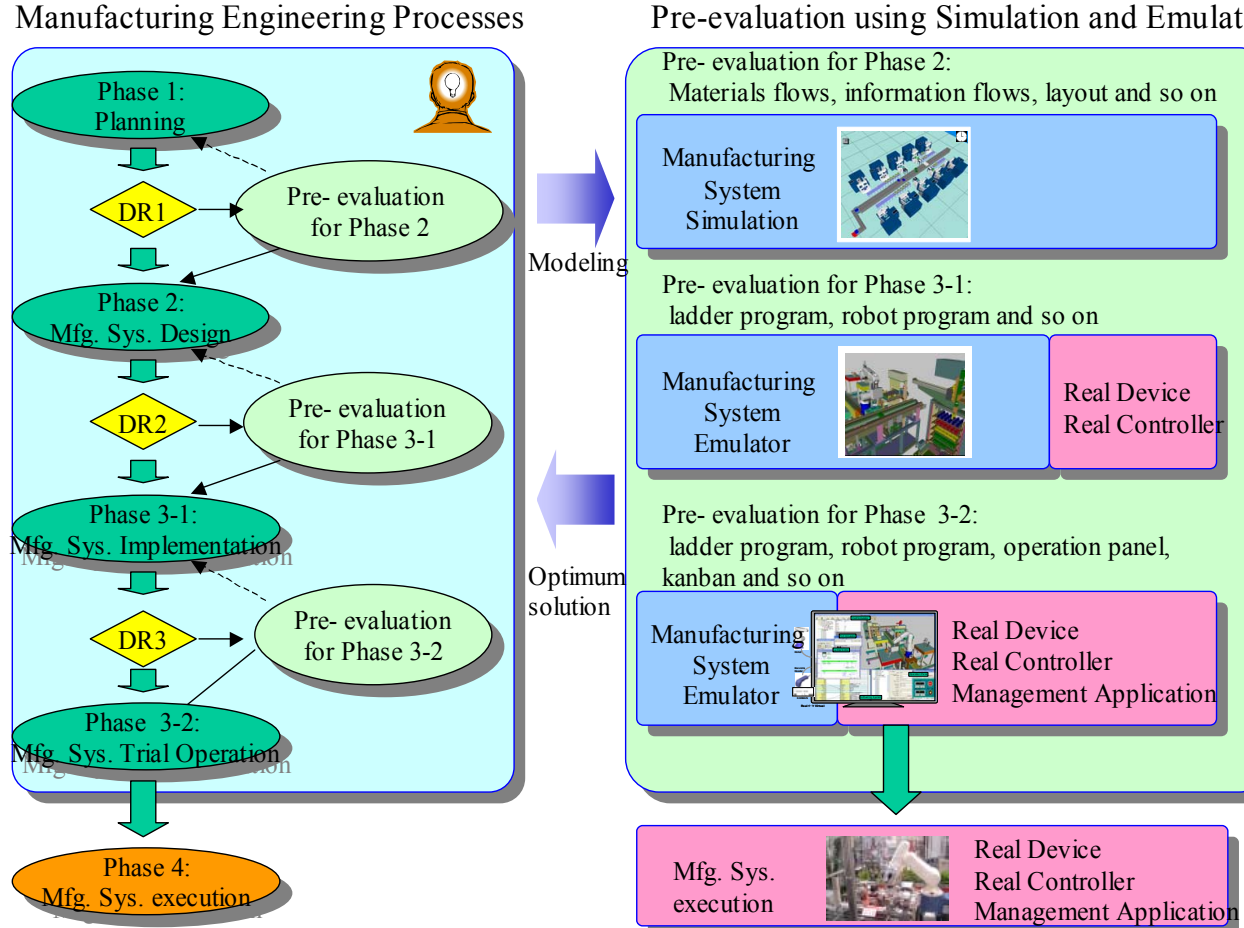

Pre- evaluation for Phase 3-2:

ladder program, robot program, operation panel, kanban and so on

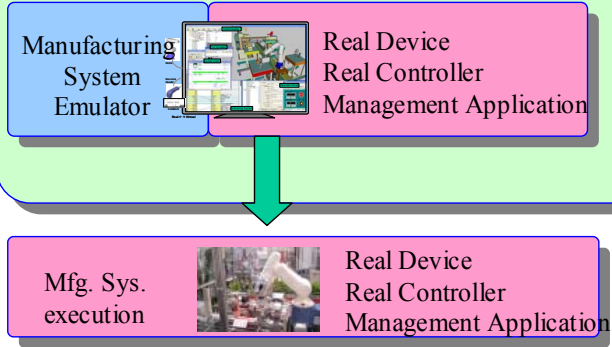

Figure 4: Proposed Manufacturing Engineering Process Using Simulation and Emulation 
MCEE realizes that the manufacturing system operates by mixing and synchronizing real equipment, real devices, real controllers, management applications, and the manufacturing system emulators. Figure 5 shows the concept of MCEE.

To realize MCEE, the following functions are necessary.

1. An emulator function to emulate manufacturing cell behaviors by using data from a real world which consists of the real equipment, real controllers, and management applications.

2. A wiring function to logically wire the real world data, the data on an emulator world implementing the emulator function, and data on manufacturing management applications.

3. A transmission function to transmit the signals and the data between the emulator world and the real world without considering the differences between the real controllers and the emulators.

4. A network interface function to connect the real controller and the emulators in a standard manner.

In order to realize these functions, we propose a manufacturing cell emulator to accomplish function 1 , and a soft-wiring system to perform function 2 . The manufacturing cell emulator emulates mechanical behavior in accordance with signals from the soft-wiring system.

Functions 3 and 4 are achieved by ORiN. ORiN (Open Resource interface for the Network / Open Robot interface for the Network) is an industrial middle-ware based on an object oriented technology (Inukai and Sakakibara 2004). It provides a standard access method to various controllers.

ORiN consists of two parts, an engine and a provider. The ORiN engine provides the application interfaces and some sophisticated common functions. The ORiN providers provide abstracted equipment models which are able to resolve the differences between abstracted equipment and real equipment. The ORiN specification defines many varieties of equipment interfaces as the providers. The applications on ORiN operate equipment through the providers via the ORiN engine.

The soft-wiring system and the manufacturing cell emulator are running on ORiN. Using ORiN, the proposed systems can become more general because of the independence from the equipment's specifications. Figure 6 shows the system architecture of MCEE. Figure 7 shows the information flows of MCEE. Figure 8 presents the ORiN architecture.

\section{THE SOFT-WIRING SYSTEM}

In actual manufacturing systems, wiring between controllers usually used hard-wiring. As MCEE is used

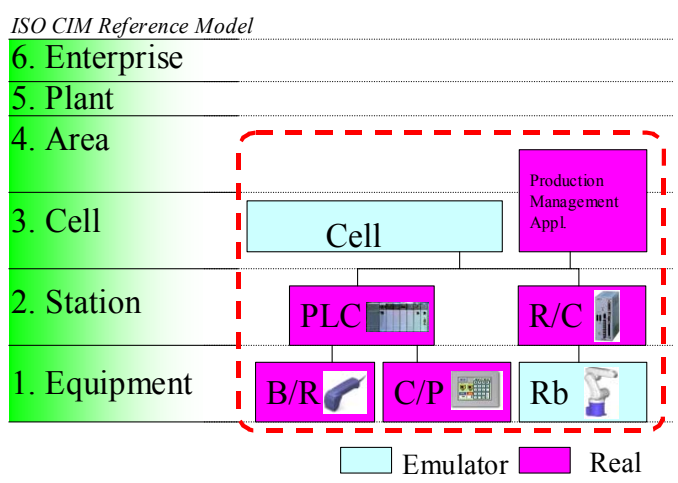

Figure 5: Concept of MCEE

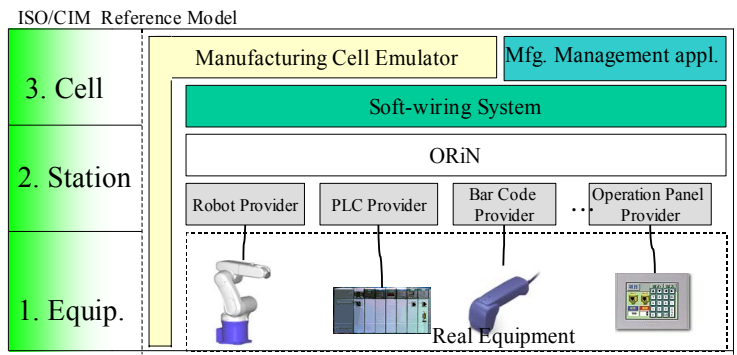

Figure 6: System Architecture of MCEE

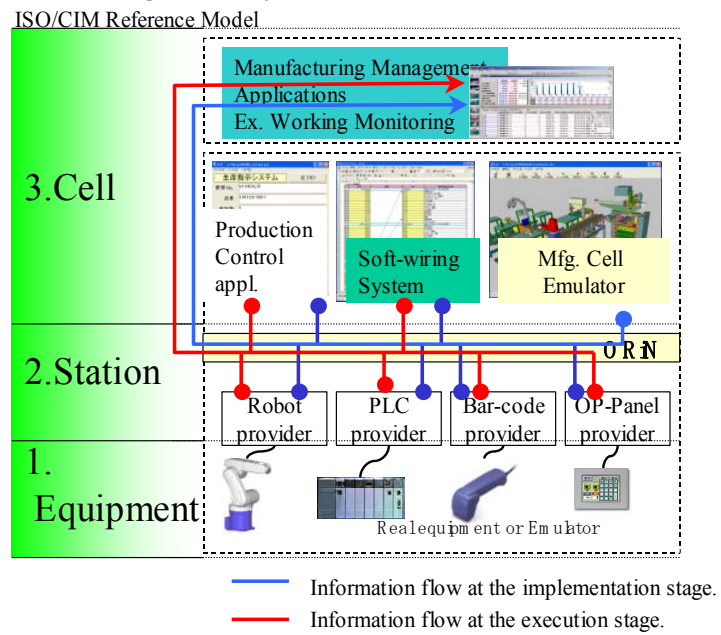

Figure 7: Information Flows of MCEE

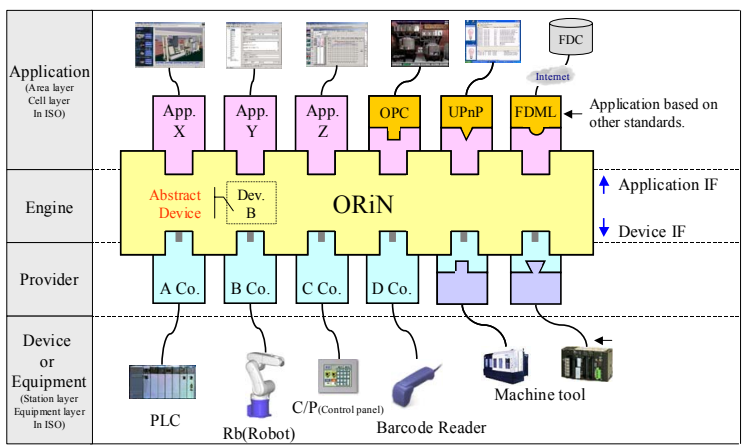

Figure 8: ORiN Architecture 


\section{Hibino and Fukuda}

before manufacturing systems are implemented, we need to evaluate the facility control programs of the controller without hard-wiring. Therefore the soft-wiring system, which logically wires the real world data, the emulator world data, and data on manufacturing management applications, is necessary. This system executes the following steps for each controller asynchronously and independently.

Step 1. Making a connection to real equipment or its emulator through ORiN.

Step 2. Observing the item value of the equipment continuously.

Step 3. Propagating the value to linked items whenever it detects a change of value.

However there are many types of data on the controller such as the integer type, the real type, the character type and so on. In order to emulate manufacturing cell behaviors using signals and data from a real world, it is necessary to transform data on the real world (or an emulator world) into utilizable data on the emulator world (or the real world). The real world means a world that consists of real equipment. The emulator world means a world that consists of equipment emulators and manufacturing cell emulation models. Therefore the soft-wiring system allows logically wiring the real world data and the emulator world data which are modeled on the emulator world. The system mainly needs to have the following functions for step 3 .

1. A function to logically wire data between the real world and the emulator world while transforming and propagating data in response to data types.

2. A function to perform the data conversions such as BCD (Binary Coded Decimal) data conversion, data masking, data type conversion such as 'String to Integer' data calculation and so on.

3. A function to filter out data which are over a limitation value as a dead band function.

4. A function to record a data transition log to evaluate a timing chart of an action sequence.

The soft-wiring system which includes four functions was developed in $\mathrm{C}++$ program language. Figure 9 shows an outline of the soft-wiring system.

\section{THE MANUFACTURING CELL EMULATOR}

In actual manufacturing systems, it is possible to evaluate facility control programs by producing materials with flow while synchronizing real equipment. As MCEE is used before manufacturing systems are implemented, it is impossible to evaluate the facility control programs in the same way. Therefore we need to have a world to emulate producing materials with flow while mixing and synchronizing real equipment, its emulators and virtual factory models.
We propose the manufacturing cell emulator to represent the world. Figure 10 shows an outline of the manufacturing cell emulator. The manufacturing cell emulator emulates manufacturing cell behaviors using signals and data from the real world through the soft-wiring system. The manufacturing cell emulator needs to have the following main functions.

1. A definition function to define behaviors for manufacturing cell emulation models.

2. An animation function to visualize $3 \mathrm{D}$ manufacturing cell emulation models by synchronizing the results of manufacturing cell behaviors.

3. A connection function to link the emulator world data to the real world data through the soft-wiring system.

We propose that the behaviors are expressed by a tree structure. One task of computer processes is defined on one node of the tree structure. Complex tasks can be defined by

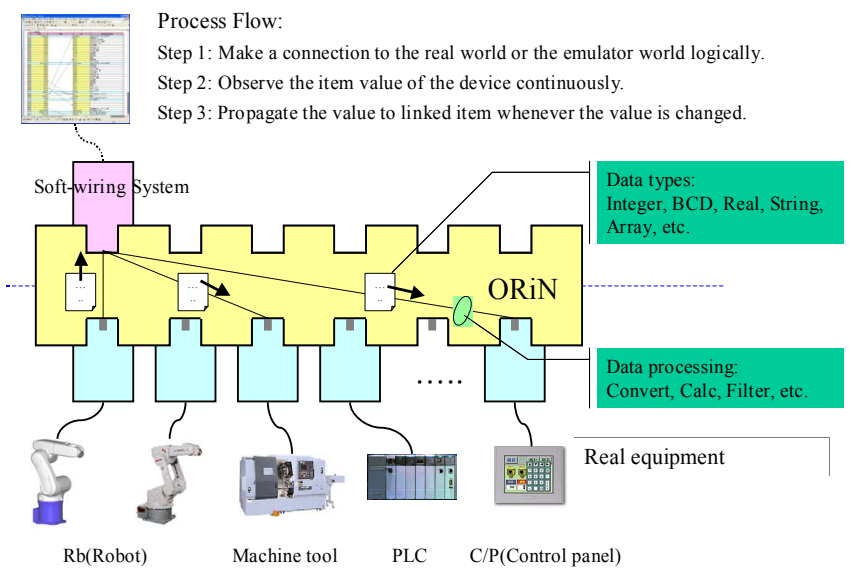

Figure 9: Outline of Soft-wiring System

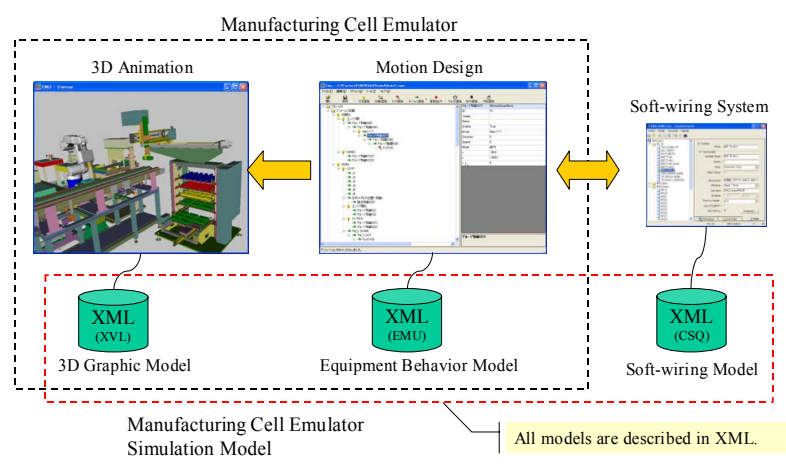

Figure 10: Outline of Manufacturing Cell Emulator

combining several nodes. And there are main four types of nodes. 


\section{Hibino and Fukuda}

1) Flow control nodes such as branch, join, and trigger.

2) A link node to link data on the emulator to the soft-wiring system

3) An animation control node to execute the animation function

4) A script node to define the details of behavior.

All nodes of the tree are executed in series or in parallel. The vertical direction is executed sequentially, and the horizontal direction is executed in parallel.

A link node 2) can refer to the real world data through the soft-wiring system. Then the manufacturing cell emulation model in the emulator world behaves in response to the referred data. The node also can output data to the real world data through the soft-wiring system. Then the equipment in the real world executes processes in response to the output data. That is, the links are bi-directional connections. For instance, when a cylinder reaches the terminal point, the cell emulator can output a signal to the softwiring system; it means that the system can output a signal to real equipment. And then the equipment which received the signal can continue to execute a program from the signal. Figure 11 shows an outline of the tree structure.

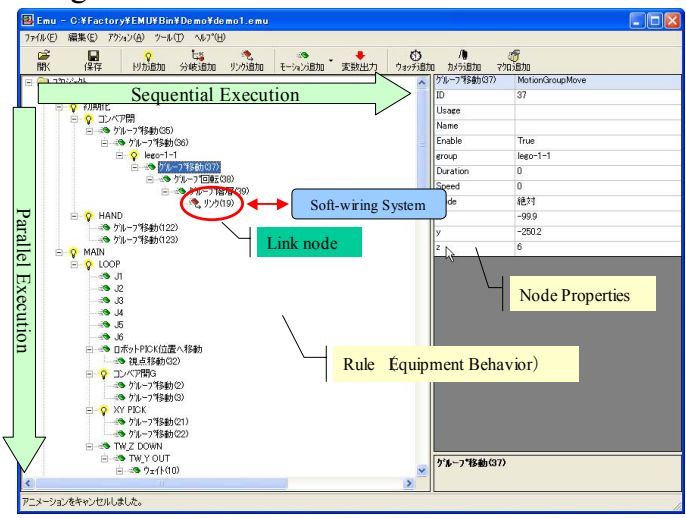

Figure 11: Outline of the Tree Structure

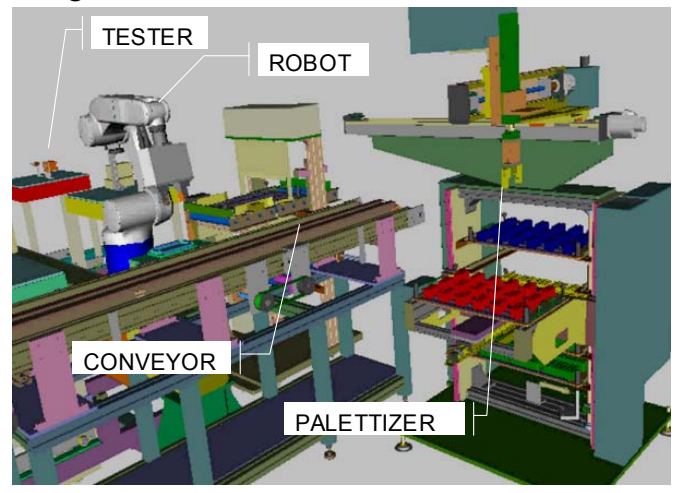

Figure 12: Case Study Models on Manufacturing Cell Emulator

\section{A CASE STUDY}

A case study was carried out using a hypothetical case of a small size of a manufacturing cell which consists of a robot, a tester, a palettizer and a conveyor. This case study consists of a robot controller, a bar-code reader and a patlite as real equipment. A PLC emulator and operation panel emulator are also connected. On the other hand, there are four systems, a production control system, manufacturing cell emulator, soft-wiring system, and working monitoring system. The production control system and the working monitoring system are real systems running in the actual manufacturing system. Figure 12 shows the case study models of the manufacturing cell emulator.

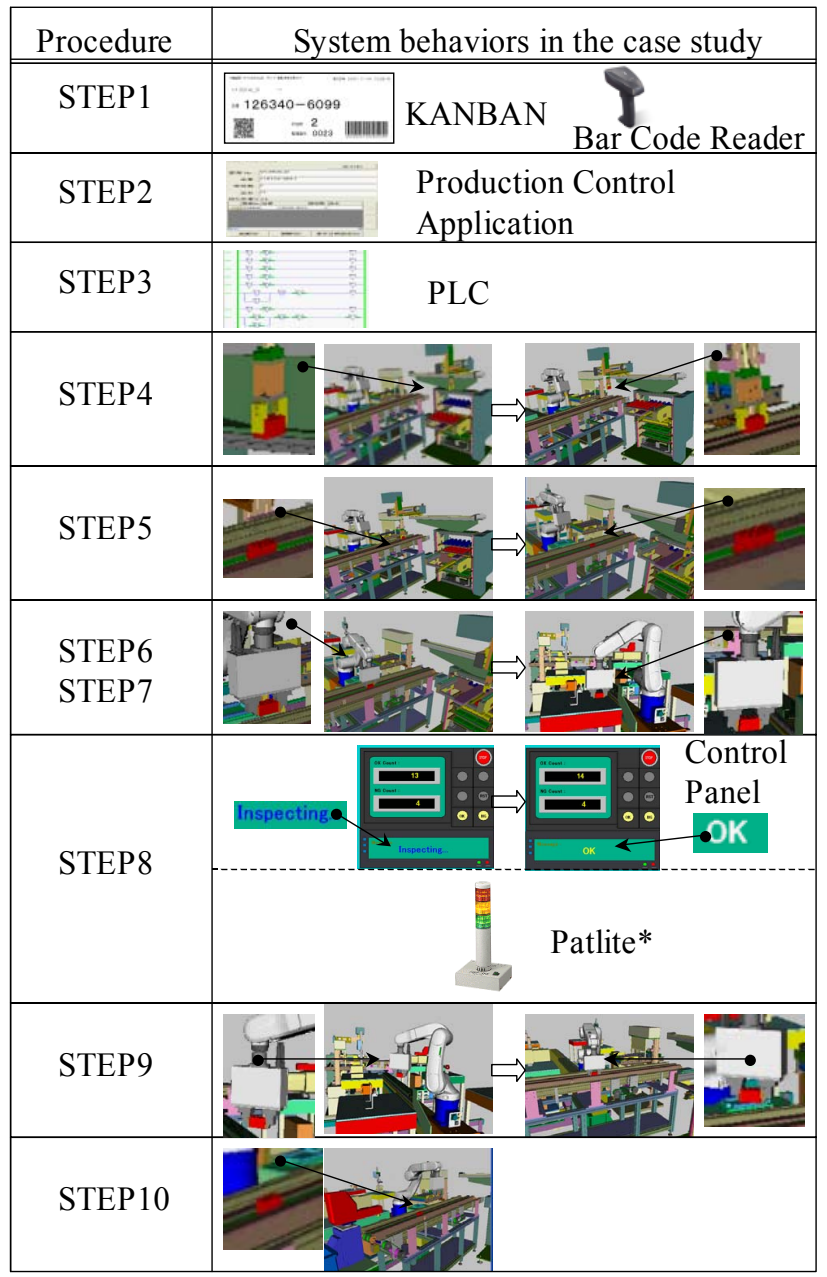

*Note:Patlite is one of the equipment which show warning signs. In this case study, for example, the color of green means the result of investigation is true.

Figure 13: Typical Procedures in this Case Study 


\section{Hibino and Fukuda}

Figure 13 presents one of the typical procedures in this case study. Figure 14 shows the system structure of this case study. Figure 15 presents one of the working monitoring applications windows which were monitored during the manufacturing cell in this case study.

1. The barcode reader receives production order information by a KANBAN.

2. The production control system receives the production order information via ORiN.

3. The production control system indicates the need to investigate the quantity of a product along with the production order information toward the PLC via the soft-wiring system.

4. The assigned product is picked up and moved to the conveyor by the palettizer in the emulator. These behaviors are controlled by the PLC via the soft-wiring system.

5. The assigned product is translated to a position in front of the robot by the conveyor in the emulator. This behavior is controlled by the PLC via the soft-wiring system.

6. The assigned product is picked up and moved to the tester by the robot. These behaviors are controlled by the PLC and the robot controller via ORiN.

7. The assigned product is investigated by the tester in the emulator.

8. Results of this investigation in the emulator are sent to the PLC via the soft-wiring system. Then the control panel displays the results via the PLC. The patlite shows warning signs along the results via the PLC.

9. The assigned product is picked up and moved from the tester to the conveyor by the robot. These behaviors are controlled by the PLC and the robot controller via ORiN.
10. The assigned product is translated to a position to the end of conveyor by the conveyor in the emulator. This behavior is controlled by the PLC via the soft-wiring system.

11. The assigned product after evaluation is eliminated in the emulator.

We confirmed that the emulator world and the real world could be combined and synchronized using the emulator, the real equipment, the real controllers, and management applications. We also confirmed that verification of the facility control programs such as a ladder program in the PLC could be confirmed by combining and synchronizing the emulator world and the real world. We also confirmed that practical usages of manufacturing management applications such as a working monitoring application could be confirmed by combining and synchronizing the emulator world and the real world.

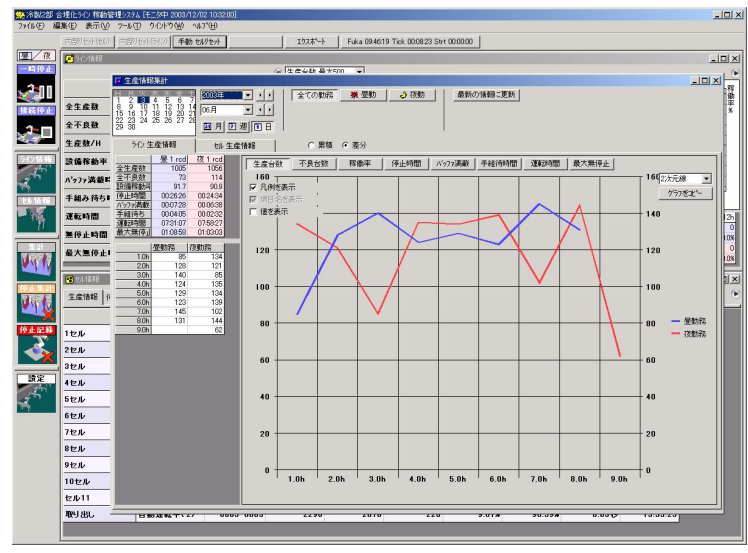

Figure 15: Working Monitor in This Case Study

Network

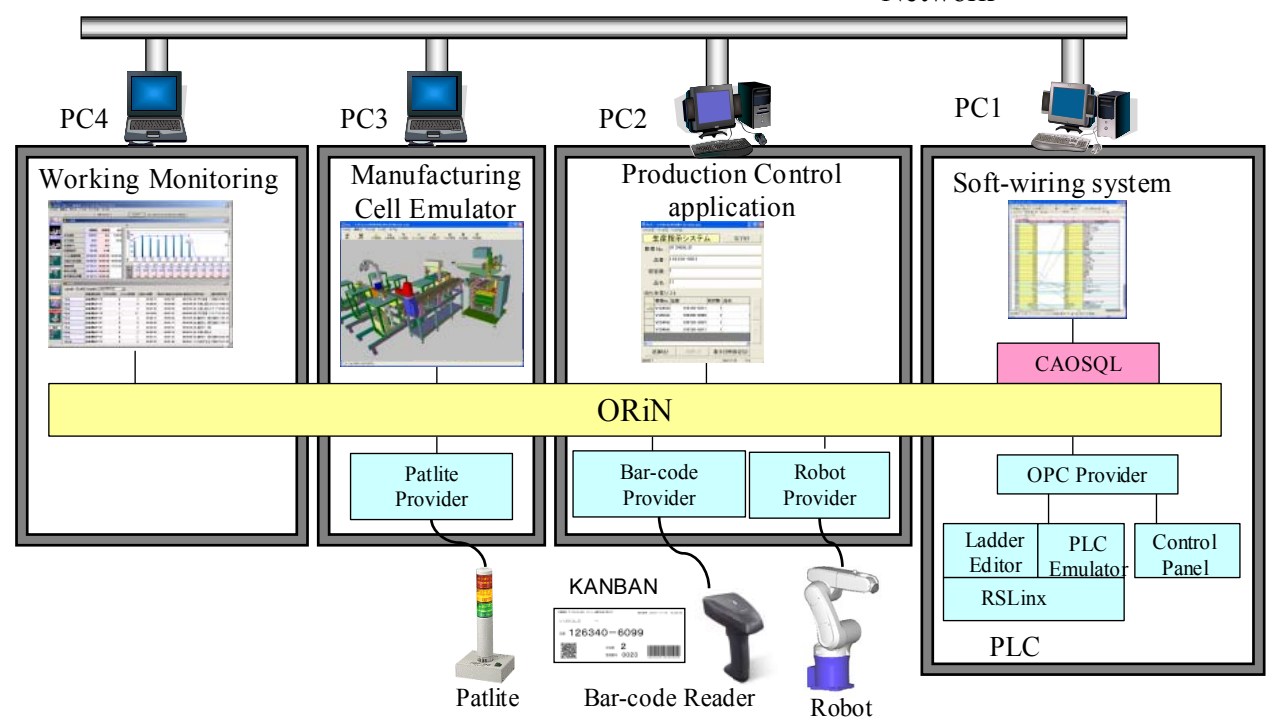

Figure 14: System Structure of This Case Study 
Through this case study, we confirmed that MCEE could be used in the implementation phase. MCEE, including the soft-wiring system and the manufacturing cell emulator and $\mathrm{ORiN}$, is a valid to provide more efficient manufacturing system implementation during the implementation phase.

\section{CONCLUSION}

In this paper, the manufacturing cell emulation environment (MCEE), which includes the soft-wiring system, the manufacturing cell emulator, and the industrial network middleware, is proposed and developed.

The results were:

1. To define terms of the manufacturing system simulation, the manufacturing system emulator, and the manufacturing system emulation.

2. To summarize the roles of the manufacturing system emulation based on our analysis for typical manufacturing engineering processes.

3. To propose MCEE realizes that the manufacturing system operates by mixing and synchronizing real equipment, real devices, real controllers, management applications, and the manufacturing system emulators under a condition where parts of the equipment, control programs, and manufacturing management applications are not provided in a manufacturing system.

4. To clarify necessary functions for a manufacturing cell emulator and a soft-wiring system in MCEE.

5. To confirm through a case study that an emulator world and a real world could be combined and synchronized using the manufacturing cell emulator and the soft-wiring system.

6. To confirm that verification of ladder programs could be confirmed by a combination of the emulator world and the real world before actual manufacturing cells are implemented.

7. To confirm that practical usages of a working monitoring application as manufacturing management applications could be verified by a combination of the emulator world and the real world before actual manufacturing cells are operated.

8. To confirm that MCEE is valid to carry out support of full production performance at product launch.

\section{ACKNOWLEDGMENTS}

This research is a part of the research project on manufacturing support systems using industrial standard technologies in JSPMI. This project was supported by funding from JKA.

This research is also a part of the research projects on efficient manufacturing system implementation based on simulation environment synchronizing real equipment and
3-D virtual factory model by IMS Idea Factory Group No.9 and No.17. These projects were supported by funding from IMS promotion center in Japan.

\section{REFERENCES}

Mehrabi, G., G. Ulsoy, and Y. Koren. 2000. Reconfigurable manufacturing systems: a key to future manufacturing. Journal of the Intelligent Manufacturing 11:403-419.

Molina, A., C. Rodriguez, H. Ahuett, J. Cortes, M. Ramirez, G. Jimenez, and S. Martinez. 2005. Nextgeneration manufacturing systems: key research issues in developing and integrating reconfigurable and intelligent machines. International Journal of Computer Integrated Manufacturing 18:525-536.

Hibino, H., and Y. Fukuda. 2006. A user support system for manufacturing system design using distributed simulation. Production Planning and Control 17:128142.

Hibino, H., T. Inukai, and Y. Fukuda. 2006. Efficient manufacturing system implementation based on combination between real and virtual factory. International Journal of Production Research 44:3897-3915.

Hibino, H.. 2007. Simulation environment for efficient manufacturing system design and implementation using network middleware ORiN and HLA. Joutnal of the Society of Instrument and Control Engineers 46:560-545. [in Japanese]

Fukuda, Y.. 2003. The state of the art for digital engineering. Journal of the Japan Society of Mechanical Engineers 106:230-233. [in Japanese]

Hibino, H., Y. Fukuda, S. Fujii, F. Kojima, K. Mitsuyuki, and Y. Yura. 1999. The development of an objectoriented simulation system based on the thought process of the manufacturing system design. International Journal of Production Economics 60:343-351.

Hibino, H., Y. Fukuda, Y. Yura, K. Mitsuyuki, and K. Kaneda. 2002. Manufacturing adapter of distributed simulation systems using HLA. In Proceedings of the 2002 Winter Simulation Conference, ed. E. Yucesan, C. Chen, J. L. Snowdon, and J. M. Charnes, 10991109. Piscataway, New Jersey: Institute of Electrical and Electronics Engineers, Inc..

Mitsuyuki, K., F. Kojima, H. Douba, Y. Fukuda, and E. Arai. 2004. Simulation to design and improve kanban system. CIRP J. Manufacturing Systems 33:200-206.

Williams, E., and H. Celik. 1998. Analysis of conveyor systems within automotive final assembly. In Proceedings of the 1998 Winter Simulation Conference, ed. D. J. Medeiros, E. F. Watson, J. S. Carson, and M. S. Manivannan, 915-920. Piscataway, New Jersey: Institute of Electrical and Electronics Engineers, Inc..

Inukai, T. and S. Sakakibara. 2004. Impact of open FA system on automobile manufacturing. Journal of the 
Automotive Engineers of Japan 58:106-111. [in Japanese]

\section{AUTHOR BIOGRAPHIES}

HIRONORI HIBINO is a Senior Researcher of the Technical Research Institute of Japan Society for the Promotion of Machine Industry (JSPMI), which is an affiliate of the Ministry of Economy, Trade and Industry in Japan. Dr. Hibino is also a Guest Professor at the Tokyo University of Agriculture and Technology concurrently. He received his BE degree from Tokyo University of Science in 1989 and his Dr. Eng. from Hosei University in 2003. His research fields are distributed simulation, manufacturing system design using simulation, manufacturing system emulation, and digital engineering. He is a member of JSME, JIMA, and JSPE.

YOSHIRO FUKUDA is a Professor of the Systems Design Department of Hosei University. He received his BS degree from Chuo University in 1971 and his Dr. Eng. from Kobe University in 1989. His main research fields are manufacturing system design, production management, manufacturing system simulation and logistics. Dr. Fukuda is a fellow of JSME. He is a member of JSME, JIMA, JSPE and IFIP5.12. He is a sector board member in IEC. 